\title{
Construcción de esculturas de hormigón con elementos procedentes del reciclado
}

\author{
Concrete sculptures construction with recycling elements
}

J.M. Saval(*), A. Maciáa ${ }^{(*)}, \underline{\text { S. Ivorra }}{ }^{(*)}$

\section{RESUMEN}

Este trabajo recoge el desarrollo conceptual, de proyecto, construcción y de ejecución de un conjunto de siete esculturas diseñadas para actuar como contenedores de otras obras de arte (17 cuadros) realizadas por el artista Jesús Soler. Este conjunto escultórico basa su mensaje en la sostenibidad y el reciclaje. Presenta, como condiciones de contorno fundamentales, la capacidad de ser montado y desmontado sin necesidad de anclar al terreno, durabilidad y protección de las obras frente a agentes meteorológicos y todo ello transmitiendo por sí mismas un mensaje medioambiental. Se describe en el documento la geometría y proporciones basadas en el "Modulor" de Le Corbusier y, por consiguiente, en la sección áurea, así como todas las técnicas constructivas aplicadas para el correcto diseño de cada obra: sistemas de encofrado, hormigones con áridos reciclados, marcos de acero corten, anclajes, etc. Finalmente se describe el primer proceso de montaje de esta obra itinerante, en el Parque del Retiro de Madrid.

$101-4$

Palabras Clave: esculturas de hormigón, reciclaje, sostenibilidad, arte y ciencia, esculturas desmontables.

\section{SUMMARY}

This paper shows the conceptual development, project, constructive and execution processes of a group of seven sculptures designed to act as containers of other art works carried out by the Spanish artist Jesús Soler. This sculptural group bases its message on the sustainability and the recycling of materials. Its fundamental boundary conditions are the capacity to be mounted and dismounted without any anchorage to the soil, durability and protection of the art work in front of meteorological agents. The sculptural group transmits by itself an environmental message. This paper describes the geometry and proportions, based on the "Le Corbusier Modulor" and the golden section, as well as all the constructive techniques applied for the correct design of each sculpture: formwork systems, concrete with recycled arid, corten steel frames, anchorages, etc. Finally the first assembly process of this itinerant sculptural work is described; it is mounted in the Parque del Retiro in the city of Madrid (Spain).

Keywords: concrete sculptures, recycling, sustainability, art and science, dismountable sculptures.

\footnotetext{
(*) Departamento de Ingeniería de la Construcción, OOPP e IU. Universidad de Alicante, Alicante (España)
} 


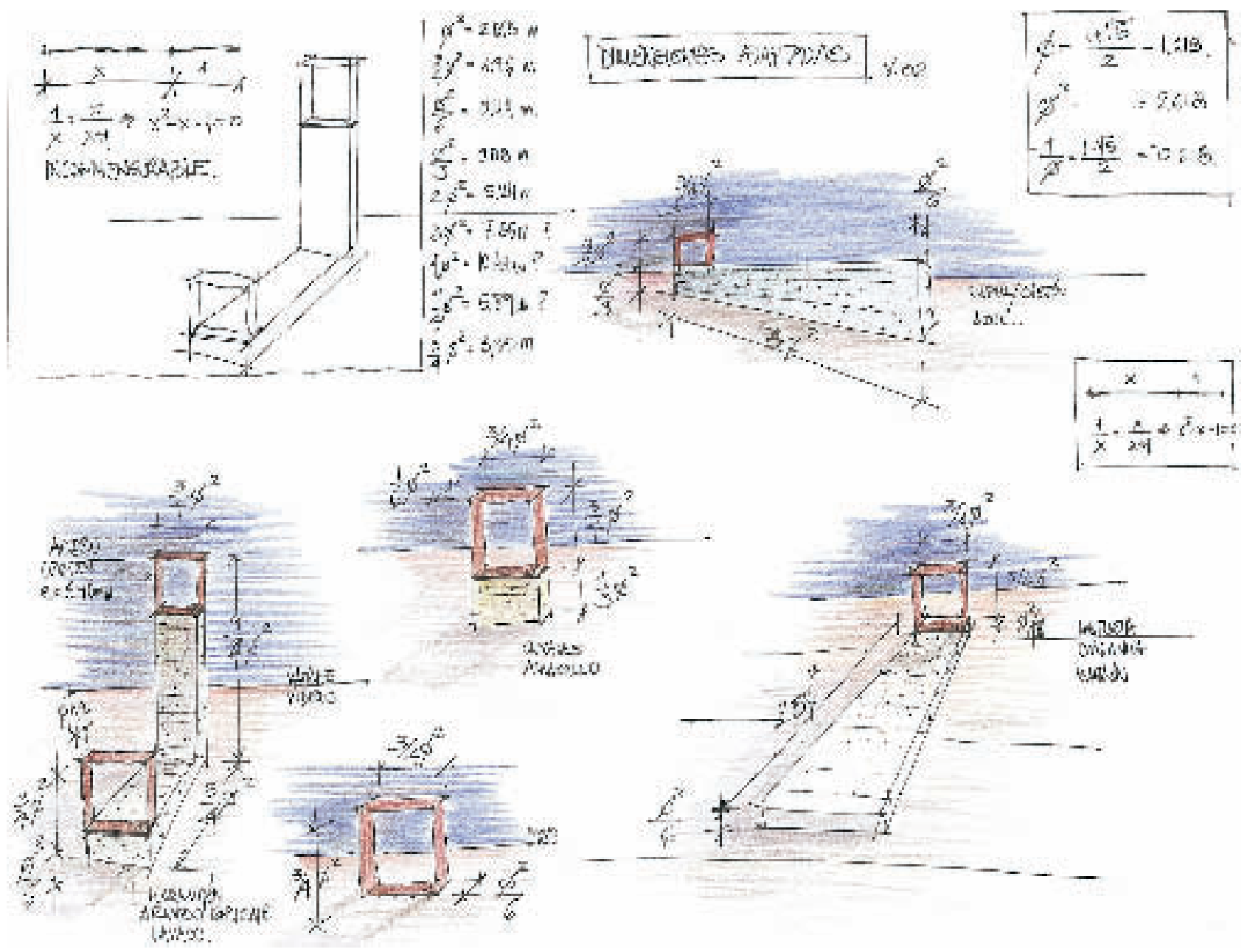

1. Bocetos de diseño en los que se incluyen las proporciones áureas.

\section{ANTECEDENTES}

La mayor parte de los trabajos científicos publicados en la actualidad en el campo del hormigón con materiales reciclados están relacionados con la incorporación de aditivos o adiciones procedentes de la recuperación de otros materiales de construcción (1), la utilización de áridos reciclados de diversa índole: elementos metálicos también recuperados de los residuos urbanos (2), o la incorporación de aditivos diversos procedentes o no de la construcción: vidrio (3), humos de Sílice, Polietileno, PVC $(4,5)$, etc. Incluso se han incorporado como aditivos al hormigón residuos provenientes de la industria del automóvil (6), elementos de circuitos electrónicos $(7,8)$, y otros tipos de residuos, incluso de origen orgánico $(9,10)$. En definitiva la búsqueda de un "hormigón verde" como lo definen Damtoft (11) y C. Meyer (12) capaz de actuar como catalizador para poner de nuevo en servicio residuos generados por la actividad humana.

En estos trabajos los objetivos prioritarios son la validación del material resultante como un hormigón, la caracterización de éste desde un punto de vista resistente/mecánico, de viabili- dad técnica y, por supuesto, su durabilidad. A pesar que el hormigón ha sido un material ampliamente utilizado para la construcción de esculturas (13), no es habitual observar la fabricación de obras de arte con hormigón y elementos procedentes del reciclaje. El trabajo que se describe en este documento es el desarrollado para construir esculturas contenedoras de otras obras de arte, formadas por elementos de hormigón fabricado con árido reciclado y elementos procedentes del reciclado de residuos urbanos que se incorporan como elementos propios del hormigón (envases de plástico, vidrio, cartón, neumáticos, ...) cuya misión es la de realzar la estética de la propia escultura. Utilizados como medio, como partes de un lenguaje capaz de transmitir, a través del arte, un mensaje social. Este hormigón no ha de presentar requisitos excesivamente exigentes respecto a su capacidad mecánica ni a su durabilidad.

A través de este conjunto escultórico se desarrolla un trabajo multidisciplinar en el que se pretende fusionar el arte con la tecnología con el fin de concienciar a la sociedad sobre la necesidad de reciclar y cuáles son las consecuencias de no reciclar. El fin del trabajo consiste en el diseño y construcción de siete 
piezas -contenedores- que permitan albergar 17 cuadros del artista Jesús Soler elaborados de igual modo con residuos y bajo la figura de una Menina. El conjunto de contenedores y cuadros viajan por España, siendo la inauguración de la primera exposición en Madrid: Madrid Recicla, el 3 de abril de 2009.

\section{DISEÑO CONCEPTUAL DE LOS CONTENEDORES}

Cada uno de los elementos escultóricos diseñados se han denominado "contenedores" por dos motivos, el primero por contener los cuadros realizados por el artista y el segundo por establecer un símil formal con contenedores de recogida de residuos urbanos: vidrio, papel y cartón, envases, tecnología, orgánicos, sólidos. Al inicio de la exposición se plantea un elemento sobre el que se instalan medios multimedia para simbolizar el no reciclado, cuyo color es el negro.

Se plantea como objetivo fundamental de cada obra el reciclado y por tanto todas las esculturas muestran que están construidas con materiales reciclados. Se decide por tanto utilizar materiales capaces de ser reciclados con facilidad: hormigón, vidrio y acero. Asimismo cada contenedor dispondrá de elementos visibles que representen claramente su tipo de residuo. Como nexo común de todos los materiales, el hormigón estará fabricado con áridos reciclados procedentes de la industria del mármol alicantina.

Estos contenedores recogerán en el interior de unos marcos de acero corten dos Meninas que, a su vez, están construidas con materiales reciclados, una de ellas denominada "viva", debido a que dispone de dos pantallas a través de las cuales se emiten mensajes medioambientales relacionadas con su tipo de residuo, y la otra, "muerta", que simboliza el no reciclaje. Las dimensiones de cada uno de los cuadros, a las que han de adecuarse las esculturas, están en torno a los $1,95 \times 1,95 \mathrm{~m}$.

Debido al carácter itinerante de la exposición y su ubicación en el exterior, los materiales escogidos han de cumplir los necesarios requisitos de durabilidad y resistencia, garantizando la estabilidad de cada escultura y la propia seguridad de cada una de las Meninas que contienen. Todas las esculturas han de ser, de igual modo, desmontables y fácilmente transportables por medios convencionales.

Una vez definido el marco de trabajo, se introduce como criterio compositivo de la obra la utilización de un nexo en las proporciones de todos los contenedores, para lo cual se realiza un estudio geométrico de las partes que componen cada contenedor utilizando el Modulor de Le Corbusier (14) (Figura 1).

Con este requisito se pretende que la obra se integre fácilmente en parques y jardines como un elemento más del mobiliario urbano: generando un lugar donde sentarse, al tiempo que facilita la visión de la obra colocándola a una altura idónea respecto a los ojos.

Tras este proceso de diseño, combinado con continuos predimensionados de las piezas, se llega a la geometría final (Figura 2) que cumple todos los requisitos planteados hasta el momento. La Tabla 1, pág. siguiente,
2. Diseño geométrico final de los contenedores. (a) Vidrio (b) Envases (c) Orgánico. (d) Sólidos. (e) papel y cartón. (f) Tecnología.
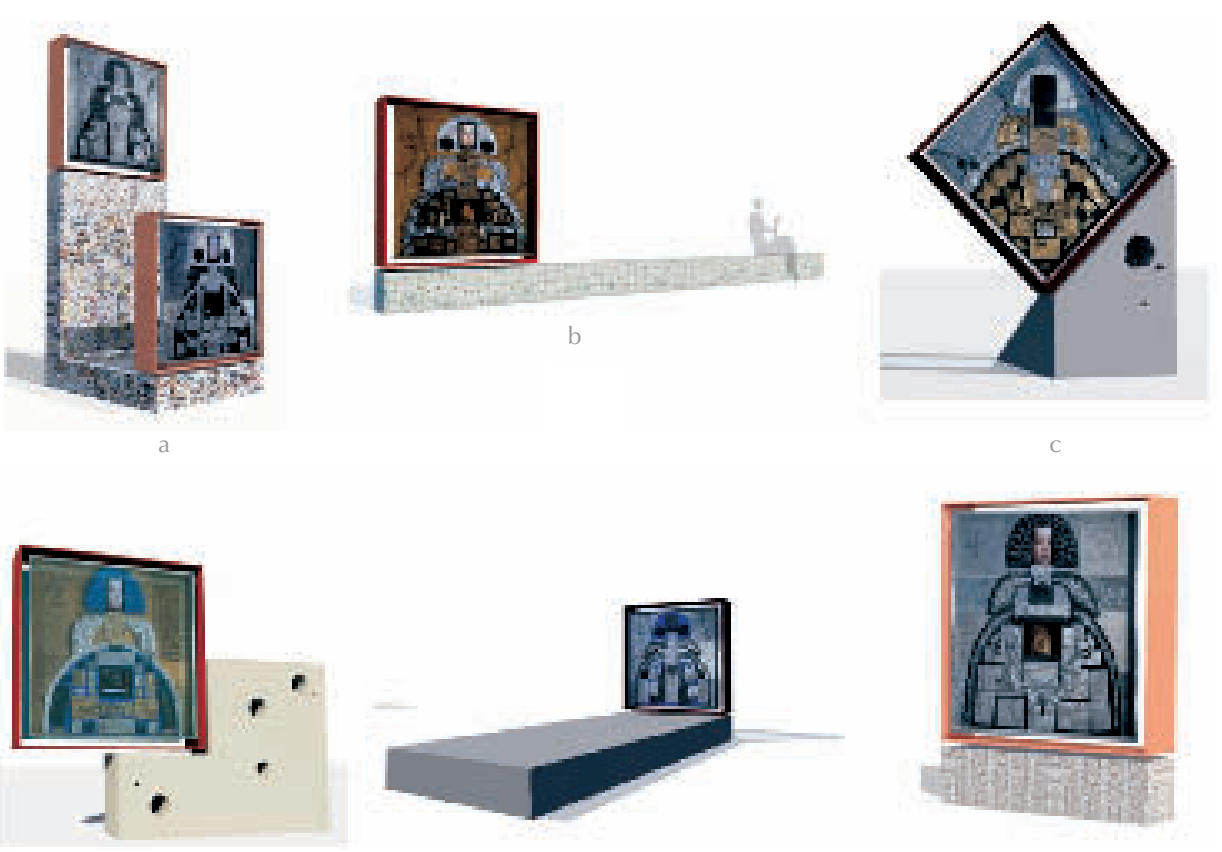
3. Modelos de elementos finitos de los contenedores más singulares. Tensiones normales en la dirección vertical-Tracción. (N/mm2) (a) Vidrio. (b) Orgánico.

4. Configuración del armado típico de todas las piezas de cada escultura. Cotas en $\mathrm{m}$.

Tabla 1

Características de cada una de las esculturas diseñadas

\begin{tabular}{|c|c|c|c|c|c|}
\hline Contenedor & \multicolumn{2}{|c|}{$\begin{array}{l}\text { Dimensiones de los } \\
\text { bloques hormigón (m) }\end{array}$} & $\begin{array}{l}\text { Dimensiones de los marcos } \\
\text { de acero corten }\end{array}$ & Color & $\begin{array}{l}\text { Peso aprox. } \\
(\mathrm{kN})\end{array}$ \\
\hline \multirow[t]{2}{*}{ Tumba } & 4 & $1,96 \times 0,43 \times 0,43$ & $1,36 \times 1,96 \times 0,43$ & \multirow{2}{*}{ Negro } & \multirow{2}{*}{74,19} \\
\hline & 3 & $2,22 \times 0,43 \times 0,43$ & & & \\
\hline \multirow[t]{2}{*}{ Vidrio } & 11 & $1,96 \times 0,43 \times 0,43$ & $1,97 \times 1,973 \times 0,43$ & \multirow{2}{*}{ Verde } & \multirow{2}{*}{112,16} \\
\hline & & & $2,023 \times 2,014 \times 0,43$ & & \\
\hline Papel y cartón & 14 & $2,02 \times 0,43 \times 0,43$ & $2,023 \times 2,021 \times 0,43$ & Azul & 137,22 \\
\hline Plástico & 8 & $1,96 \times 0,43 \times 0,43$ & $2,018 \times 2,217 \times 0,43$ & Amarillo & 78,98 \\
\hline Tecnología & 2 & $1,96 \times 0,43 \times 0,43$ & $1,96 \times 1,975 \times 0,43$ & Rojo & 24,62 \\
\hline Orgánicos & 2 & $*$ & $1,96 \times 1,96 \times 0,43$ & Gris & 44,25 \\
\hline \multirow[t]{2}{*}{ Sólidos } & 2 & $1,96 \times 0,43 \times 0,43$ & $2,139 \times 2,053 \times 0,43$ & \multirow{2}{*}{ Blanco } & \multirow{2}{*}{33,68} \\
\hline & 2 & $1,96 \times 0,43 \times 0,43$ & & & \\
\hline
\end{tabular}

* Contenedor de residuos orgánicos formado por dos piezas, una con forma de prisma cuya base es un triángulo rectángulo con catetos de 1,41 m, y una altura del prisma de 0,43 m. La otra pieza está compuesta por dos elementos adosados, un bloque de $1,41 \times 0,86 \times 0,43 \mathrm{~m}$ y un tetraedro rectangular de $1,41 \times 1,41 \times 0,86 \mathrm{~m}$.

\section{ANÁLISIS Y DIMENSIONADO RESISTENTE DE LAS PIEZAS}


muestra características generales de cada contenedor.

Siguiendo el mismo proceso de diseño que con las piezas de hormigón, se han diseñado ocho marcos de acero S275 para albergar los cuadros del artista Jesús Soler. Estos elementos metálicos han sido oxidados mediante ácido clorhídrico y posteriormente barnizados con pintura antioxidante para dar un aspecto exterior similar al del acero corten.
Para el proceso de análisis y dimensionado de las piezas se ha seguido el procedimiento clásico de cualquier estructura, en el cual ha existido una continua retroalimentación positiva entre los diversos técnicos con el fin de llegar a una solución óptima desde los puntos de vista estético, artístico y técnicoconstructivo.

Desde el primer momento se han planteado los siguientes requisitos de diseño:

- Capacidad de todos los contenedores de ser autoestables tanto para peso propio como para la acción del viento sin necesidad de anclar en el terreno.

- No trasmitir al terreno tensiones superiores a los 0,25 $\mathrm{MPa}$.

- Estructuras desmontables con elementos unitarios de peso no superior a $25 \mathrm{kN}$.

- Durabilidad tanto a agentes meteorológicos como vandálicos.

Cada una de las piezas se ha estudiado de forma independiente, así como cada uno de los elementos que la componen, tanto en su ubicación temporal como en su fase de montaje y desmontaje.

Dentro de las acciones consideradas en el diseño, ha sido la acción del viento la que 
más ha influido en su configuración definitiva. Este valor es una incógnita debido a que la ubicación de las esculturas no es fija, ya que se proyecta para ser itinerante por toda España, pudiendo ubicarse en situaciones expuestas. Se han considerado los datos de la carga de viento obtenidos del Código Técnico de la Edificación (15) (Tabla 2).

En los contenedores de residuos de tecnología, envases y residuos sólidos, esta acción ha sido decisiva a la hora de considerar un aumento en la dimensión de la base para poder aumentar la capacidad de absorber el momento de vuelco. Para ello se ha introdu-

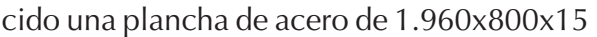
$\mathrm{mm}^{3}$. La instalación de este elemento, integrado en el conjunto escultórico, permite garantizar la estabilidad de cada estructura frente a la acción del viento.

Junto con modelos sencillos de equilibro, se han utilizado técnicas más refinadas para los contenedores con geometría más compleja frente a posibles problemas de vuelco, transmisión de cargas excesivas al terreno, o incluso zonas de concentración de tensiones. Se han realizado varios modelos numéricos basados en el método de los elementos finitos con el programa informático SAP2000 ${ }^{\mathrm{TM}}$ (16), (Figs. 3a y b). A través de los resultados proporcionados por estas simulaciones se han se han desarrollado planos constructivos y de taller incluyendo la ubicación uno por uno de cada uno de los residuos existentes en las piezas (Figura 5a). Se han creado los negativos de cada cara de cada elemento y posteriormente se han construido unos preencofrados en los que se han adherido los residuos atendiendo al negativo de cada pieza diseñada. Estos pre-encofrados están formados por planchas de policarbonato de $4 \mathrm{~mm}$ de espesor (Figura 5b).

Posteriormente se han utilizado sistemas de encofrado convencionales de pilares para edificación tipo "Alisply pilares" en los que se ha dispuesto la armadura, con separadores de $3 \mathrm{~cm}$, junto con los pre-encofrados que contienen los residuos.

En la Figura 6, pág. siguiente, pueden observarse unos tubos pasantes de $\varnothing 20$ de PVC, perpendiculares a la armadura longitudinal, colocados en la fibra neutra de la sección.

El objetivo de estos encofrados perdidos es el generar un hueco, a través del cual puedan introducirse unas barras metálicas para el posterior izado de las piezas en las fases de montaje y desmontaje. Las dimensiones de estos huecos han sido condicionadas por la propia fase de cálculo de los elementos auxiliares de izado.

Tabla 2

Evaluación de la acción de viento de cálculo

Zona eólica C (velocidad básica del viento de $29 \mathrm{~m} / \mathrm{s}$ )

Grado de aspereza del entorno tipo IV

Presión dinámica: $0,53 \mathrm{kN} / \mathrm{m}^{2}$

Coeficiente de exposición: 1,34

Coeficiente de presión: 1,3

Coeficiente corrector para periodo de retorno de 10 años: 0,9

Acción de viento de cálculo: 0,91 kN/m²

podido ajustar las geometrías y armados de cada uno de los elementos individuales que los componen, así como elementos de refuerzo interior que cohesionan todas las piezas.

Con respecto al dimensionado de cada una de las piezas de cada contenedor, se han considerado diversas hipótesis, entre ellas las condiciones de contorno existentes en las fases de montaje y desmontaje; que han resultado ser las más exigentes en la mayor parte de los casos. Por facilidad constructiva se han unificado todos los armados disponiéndose de jaulas como las que se presentan en la Figura 4.

\section{PROCESO DE CONSTRUCCIÓN}

Todo el diseño de las piezas se ha realizado sobre plano, de forma gráfica, para lo que
5. (a) Planos de detalle -en negativo-de la ubicación, tamaño y colo de los residuos en el contenedor de residuos de vidrio (cotas en $\mathrm{m}$ ) (b) Detalle de la colocación de los residuos de vidrio en el pre-encofrado de policarbonato.
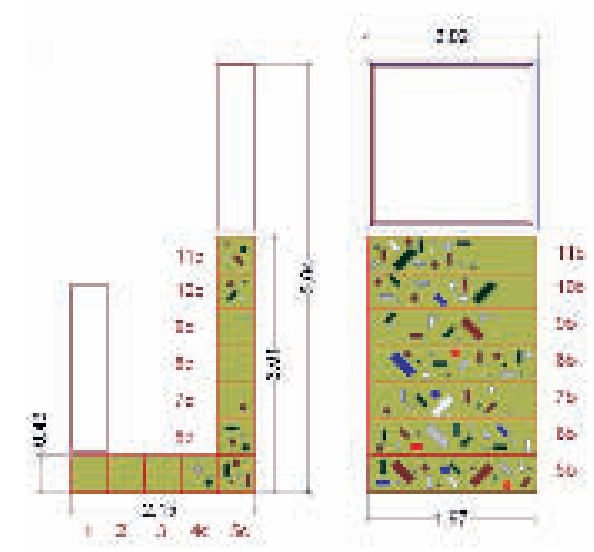

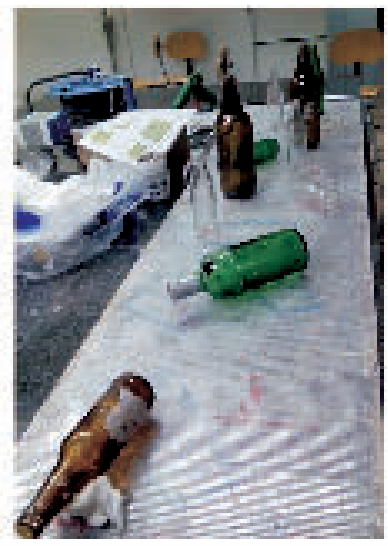




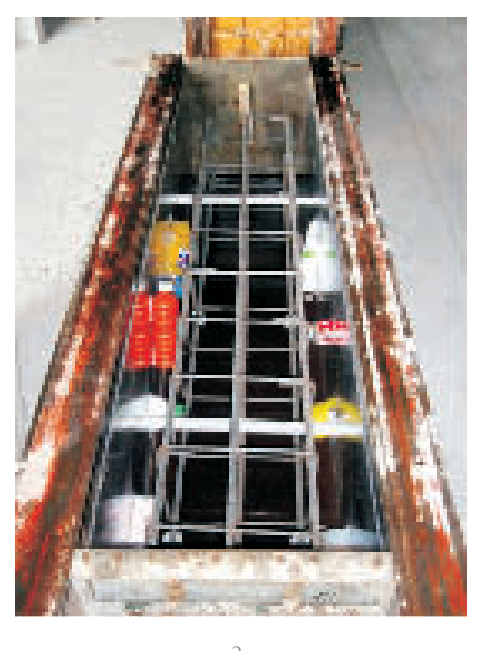

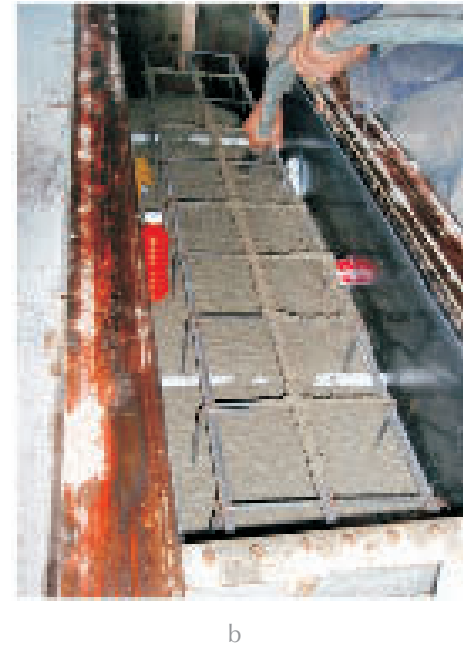

6. (a) Colocación de los pre-encofrados de policarbonato en el interior de los encofrados metálicos. (b) Vertido y vibrado del hormigón de cada pieza de hormigón.
Dado que en la fase de diseño se optó por dejar visto el árido reciclado y el propio residuo, se ha previsto un recubrimiento elevado para la posterior fase de corte de cada pieza en la que se eliminan entre 2 y $4 \mathrm{~cm}$ de la capa exterior, dejando perfectamente planas las caras de los elementos. La fabricación de estas piezas se realizó en invierno, por lo que tras el hormigonado en la planta de hormigón prefabricado se dejó curar a temperatura ambiente. Tras diez días de curado las piezas se trasladaron a una planta de corte de mármol (Fig. 7a), con el fin de eliminar una capa de entre 2-4 cm de hormigón dejando ver el árido y los residuos (Fig. 7b). En una siguiente fase y con el fin de dotar a cada uno de los contenedores del color propio del residuo al que representan se ha procedido a teñir cada uno de ellos con tinte universal diluido en agua (Figura 8), introduciendo detalles de acabados con pintura acrílica y lacas. Finalizado este proceso, todas las obras se hidrofugaron y barnizaron con el fin de dotarlas de una mayor durabilidad frente a actos vandálicos.

Para la fabricación de los marcos de acero se han utilizado perfiles L100.50 y chapas de diversos espesores de acero S275. La disposición constructiva de los perfiles puede observarse en la Figura 9. Esta configuración permite introducir láminas de espejo en su interior generando una ilusión óptica al espectador, en ella se presenta el cuadro flotando en el aire, separado del propio marco exterior. La conexión de cada marco a los bloques de hormigón se ha realizado mediante barras de acero corrugadas de $\varnothing 25$ roscadas, unidas al bloque mediante resina epoxi con una longitud de anclaje de $65 \mathrm{~cm}$ y al marco mediante doble tuerca de M20. Una vez ejecutado cada marco, se ha procedido a oxidarlo mediante una dilución de ácido clorhídrico en agua, acelerando la oxidación mediante un riego continuo, tras lo cual se ha procedido a barnizar cada marco con tratamiento específico para evitar el contacto del acero con el oxígeno y por tanto frenar el aumento de la oxidación.

\section{PROCESO DE MONTAJE}

Con el fin de que el proceso de montaje y desmontaje esté lo más optimizado posible se ha desarrollado un protocolo de trabajo para la obra completa y de cada escultura por separado. Este proceso se describe en las siguientes fases:

1. Replanteo de la ubicación de cada escultura para mantener la composición del conjunto.

2. Construcción, sobre una lámina de PVC, de una base de mortero pobre para poder nivelar las irregularidades del terreno.

3. Acopio de las piezas de cada escultura en el entorno de su ubicación.

4. Montaje de piezas de hormigón de cada contenedor por el siguiente orden: no reciclaje, papel y cartón, envases, tecnología, sólidos, orgánico y vidrio. Este orden, de menor a mayor complejidad, permite el desarrollo superpuesto de las siguientes fases en los contenedores más sencillos.

5. Montaje de marcos.

6. Instalación eléctrica

7. Colocación de Meninas. Pruebas de iluminación.

8. Colocación de vidrios.

A través de este protocolo y con un equipo de seis personas, el montaje final de toda la exposición es posible realizarla en dos jornadas de trabajo.

En la fase de montaje se introducen una serie de perfiles de acero entre las distintas piezas (Fig. 10a) con el fin de garantizar que el conjunto trabaje de forma solidaria frente a las acciones consideradas en el cálculo.

Con el mismo fin se unen los bloques de hormigón con anclajes individuales $\varnothing 16$ atornillados. Tras la finalización completa del montaje, la Figura 11, pág. 34, muestra el estado final de las piezas el día de su primera inauguración en el Parque del Retiro de Madrid el día 3 de abril de 2009.

\section{CONCLUSIONES}

Este artículo presenta la fase proyectual y constructiva de un conjunto escultórico único en el que se entrelazan los conceptos de la sostenibilidad, el medio ambiente y el reciclado. La obra en sí misma actúa como contenedor con la obra del artista español Jesús Soler, transmitiendo de forma visual, táctil y multimedia el mensaje conceptual del conjunto. 

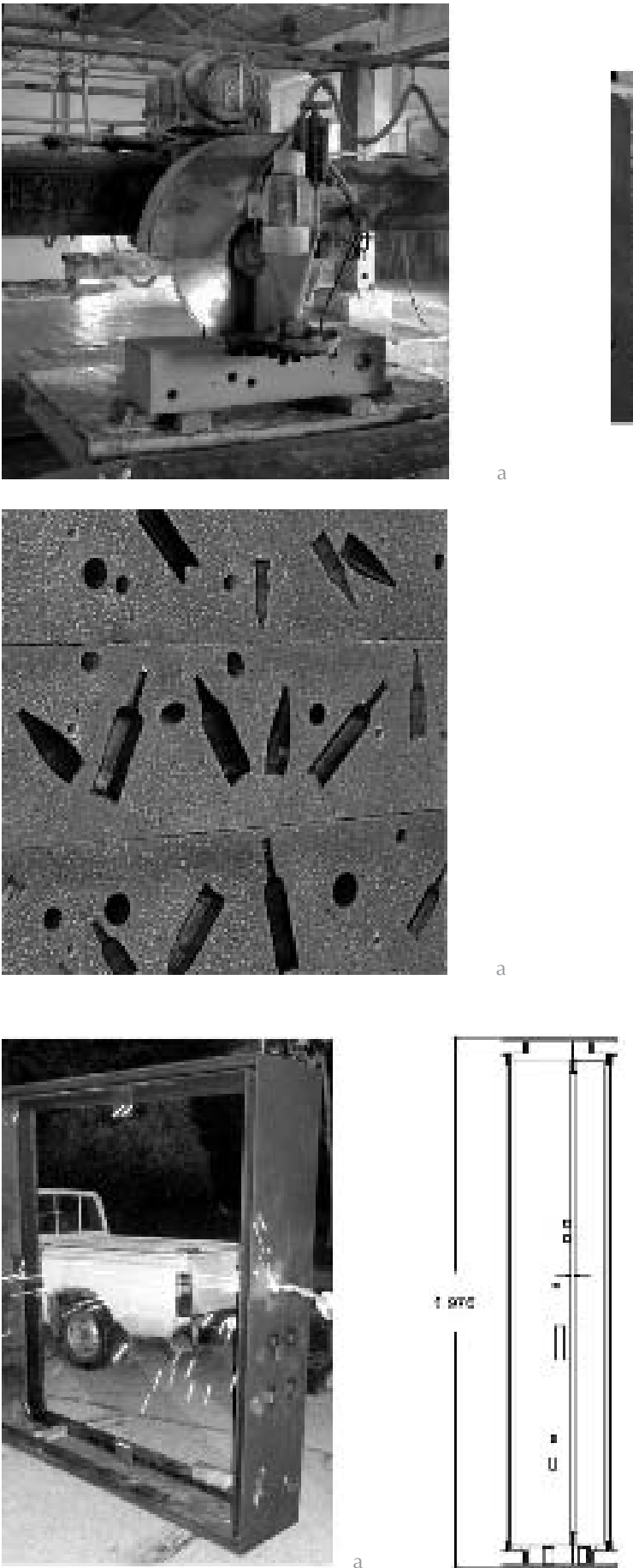

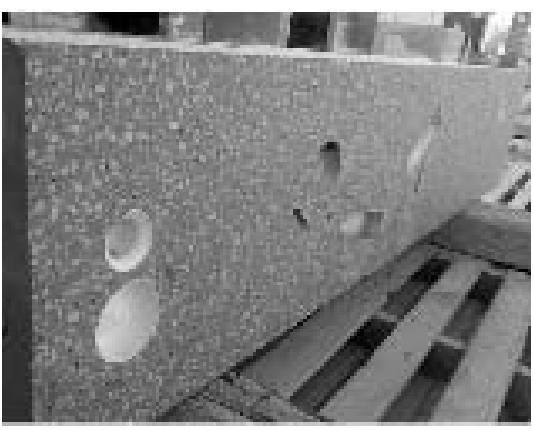

b
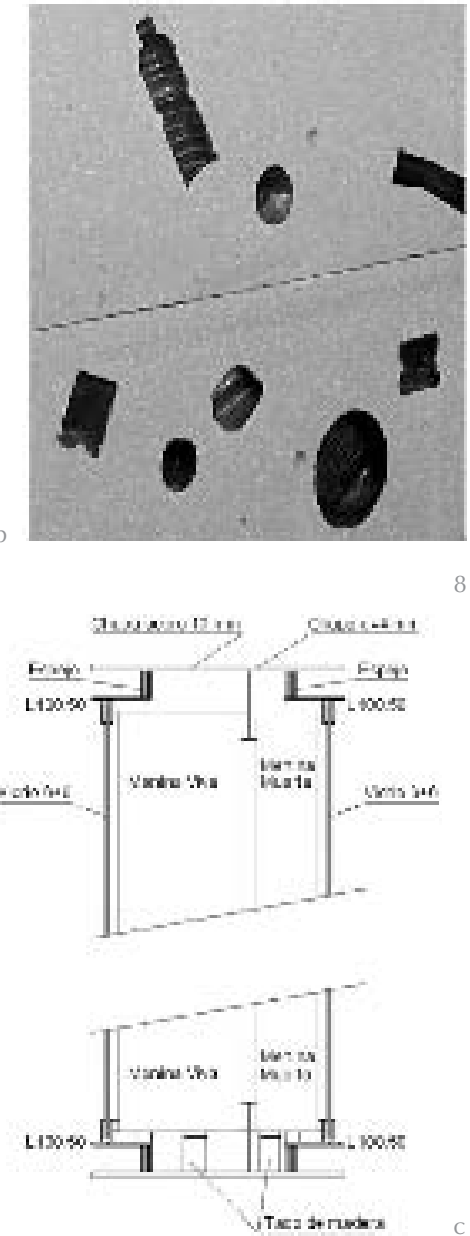

7. (a) Pieza de hormigón en el interior de la máquina cortabloques de mármol. (b) Árido visto en pieza ya cortada.

8. Detalle del teñido y lacado. (a) Residuos de vidrio. (b) Envases de plástico.

9. (a) Marco de acero prácticamente acabado. (b) Vista lateral de uno de os marcos de acero corten (c) detalles constructivos de cada marco. Cotas en $\mathrm{m}$.

10. (a) Tubos de acero para solidarizar los bloques de hormigón. (b) Anclajes atornillados para evitar el movimiento independiente de los perfiles.
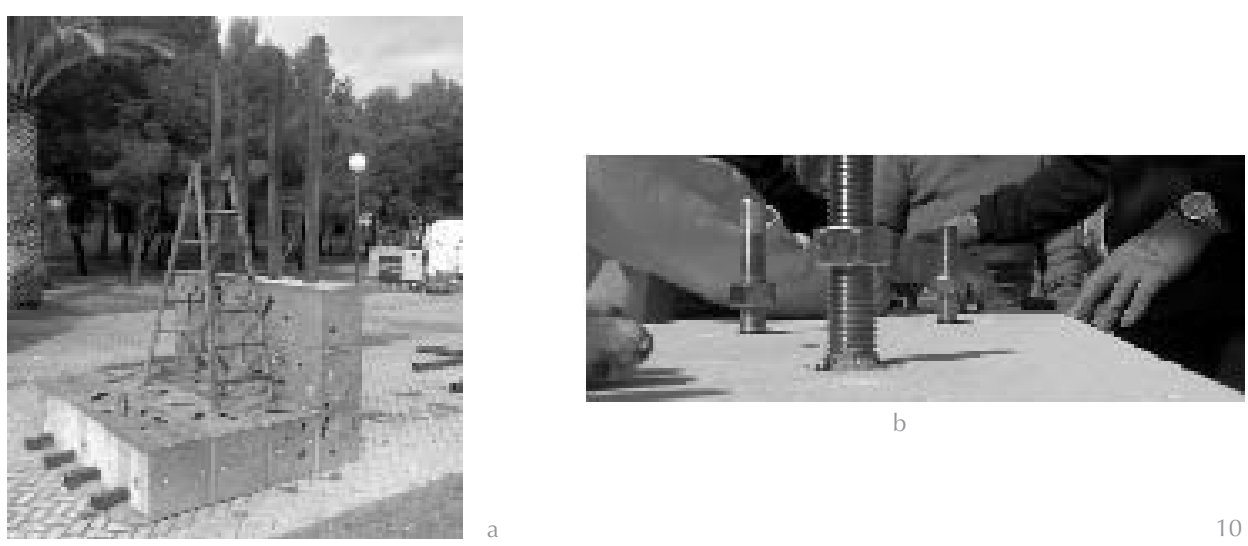
11. Contenedores construidos. (a) Vidrio (b) Envases. (c) Orgánico. (d) Sólidos. (e) Papel y cartón. (f) Tecnología.

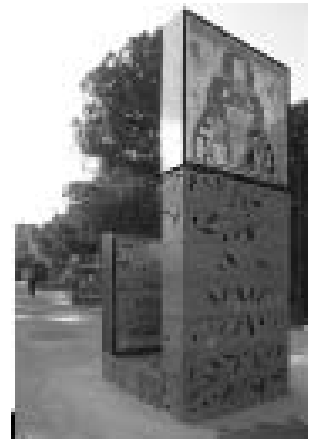

a

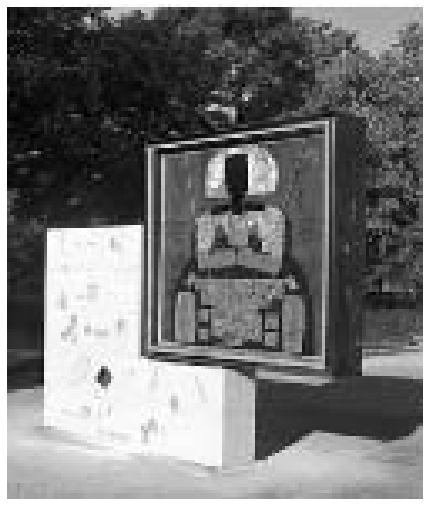

d
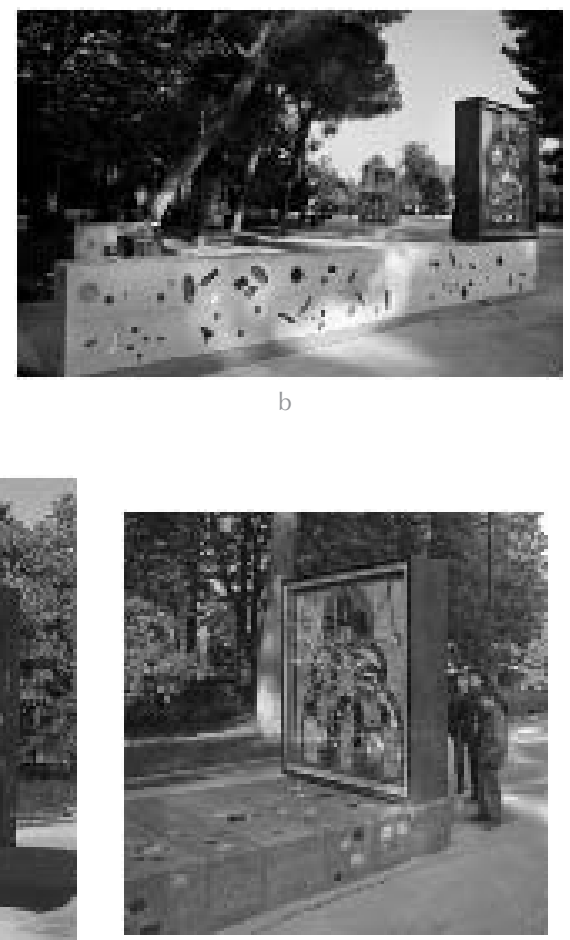

e

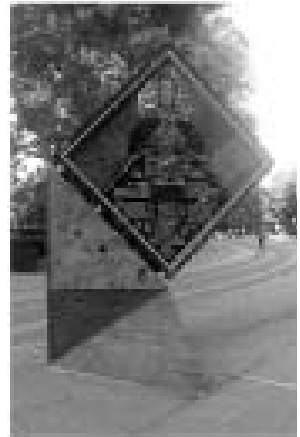

C

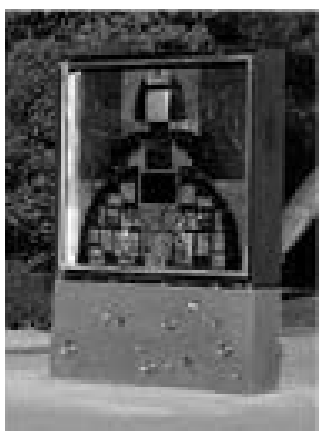

\section{BIBLIOGRAFÍA}

(1) Vivian W.Y. Tam (2009): Comparing the implementation of concrete recycling in the Australian and Japanese construction industries, Journal of Cleaner Production, Volume 17, Issue 7, Present and Anticipated Demands for Natural Resources: Scientific, Technological, Political, Economic and Ethical Approaches for Sustainable Management, pp. 688-702.

(2) Aiello, M.A., Leuzzi, F., Centonze, G., Maffezzoli, A. (2009): Use of steel fibres recovered from waste tyres as reinforcement in concrete: Pull-out behaviour, compressive and flexural strength. Waste Management, 29 (6), pp. 1960-1970.

(3) Taha, B., Nounu, G. (2008): Properties of concrete contains mixed colour waste recycled glass as sand and cement replacement. Construction and Building Materials, 22 (5), pp. 713-720.

(4) Siddique, R., Khatib, J., Kaur, I. (2008): Use of recycled plastic in concrete: A revie. Waste Management, 28 (10), pp. 1835-1852.

(5) Choi, Y.W., Moon, D.J., Kim, Y.J., Lachemi, M.: Characteristics of mortar and concrete containing fine aggregate manufactured from recycled waste polyethylene terephthalate voltees. Construction and Building Materials, Article in Press.

(6) Péra, J., Ambroise, J., Chabannet, M. (2004): Valorization of automotive shredder residue in building materials. Cem. Concr. Res., 34 (4), pp. 557-562.

(7) Cui, J., Forssberg, E. (2003): Mechanical recycling of waste electric and electronic equipment: A review. Journal of Hazardous Materials, 99 (3), pp. 243-263.

(8) Guo, J., Guo, J., Xu, Z. (2009): Recycling of non-metallic fractions from waste printed circuit boards: A review. Journal of Hazardous Materials, Article in Press.

(9) Phair, J.W. (2006): Green chemistry for sustainable cement production and use. Green Chemistry, 8 (9), pp. 763-780.

(10) Rübner, K., Haamkens, F., Linde, O. (2008): Use of municipal solid waste incinerator bottom ash as aggregate in concrete Quarterly. Journal of Engineering Geology and Hydrogeology, 41 (4), pp. 459464.

(11) Damtoft, J.S., Glavind, M. Petersen, C. M. (2001). Background to the establishment of the Danish Centre for Green Concrete. Proceedings of CANMET/ACI International conference, San Francisco, USA.

(12) Meyer, C., (2009). The greening of the concrete industry, Cement and Concrete. Composites, Article in Press.

(13) Molina, J. (1996) El hormigón como materia moldeable en la construcción de esculturas. Informes de la construcción, Vol. 48, №. 443, 1996, pp. 35-42.

(14) Le Corbusier. Le Modulor and Modulor 2. Birkhäuser Basel; 1 edition, 2004.

(15) Código Técnico de la Edificación. Ministerio de Vivienda, Gobierno de España. Marzo, 2006.

(16) SAP2000 ${ }^{\text {TM }}$ CSI Analysis Reference Manual ver. 11. Computers and Structures, Inc. Berkeley, California, USA, 2007. 